\title{
Interpretations of Quantum Mechanics and Emptiness
}

\begin{abstract}
Michele Caponigro* and Ravi Prakash ${ }^{\dagger}$
Abstract

The underlying physical reality is a central notion in the interpretations of quantum mechanics. The a priori physical reality notion affects the corresponding interpretation. This paper explore the possibility to establish a relationship between philosophical concept of physical reality in Nagarjuna's epistemology (emptiness) and the picture of underlying physical reality in Einstein, Rovelli and Zeilinger positions. This analysis brings us to conclude that the notion of property of a quantum object is untenable. We can only speak about relational property of the object. On this basis, we are stimulated to build a new ontology of underlying physical reality: a relational ontology. Finally, we argue that Nagarjuna's view is comparable with Rovelli's interpretation of quantum mechanics. These views eliminate the privileged role of the observer.
\end{abstract}

Key Words: Interpretations of QM, Einstein, Rovelli, Zeilinger, Nagarjuna, Madhyamika, relational ontology

NeuroQuantology 2009; 2: 198 - 203

\section{Introduction}

The abstract mathematical structure of the Lorentz transformations was deduced through simple physical principles. Thanks to the existence of these physical principles we do not have a significant debate on the interpretation of the theory of special relativity. In quantum mechanics, the absence of elementary physical principles has determined a broad discussion about the interpretation of the theory. Historically, the understanding of the mathematical structure of quantum mechanics (QM) went trough various stages. Very briefly, the Copenhagen interpretation assumes two processes influencing the wavefunction, namely, (i) its unitary evolution according to the Schrödinger equation, and (ii) the process of measurement. Quantum theory is problematic in

Corresponding author: Michele Caponigro

Address: * University of Bergamo - via Salvecchio 19 - 24129

Bergamo - Italy and ${ }^{\dagger}$ Central Institute of Psychiatry, India

e-mail: drravi2121@gmail.com

ISSN 13035150 the sense that it is incomplete and needs the notion of a classical device measuring quantum observables as an important ingredient of the theory. Due to this, one tends to accept that there exist two worlds: the classical one and the quantum one. In the classical world, the measurements of classical observables are produced by classical devices. In the framework of standard theory, the measurements of quantum observables are produced by classical devices, too. The Copenhagen interpretation postulates that every measurement induces a discontinuous break in the unitary time evolution of the state through the collapse of wave function, the nature of the collapse is not at all explained. Some physicists argue (Fuchs 2000; 2002; Bub 2004) that an interpretation is nothing more than a formal equivalence between a given set of rules for processing experimental data, thus suggesting that the whole exercise of interpretation is unnecessary. 


\section{The measurement problem}

\section{Quantum system}

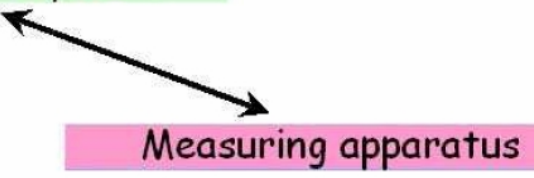

Figure 1. What is a measurement apparatus?

This point of view is rather pragmatic. Nevertheless, many physicists are not only interested in the pragmatic aspects, but also want to understand nature in a deeper conceptual level. Besides, a deeper understanding of nature in the conceptual level may also induce a new development of pragmatic aspects. Thus, the conceptual understanding of physical phenomena is also an important aspect of physics and cannot be viewed as simply epistemological problems. In the Copenhagen interpretation the state vector represents our knowledge of the system, not its physics. The main support of this interpretation is that measurement process is an interaction between system and apparatus. This interpretation divides the world in apparatus and system but the theory tells us nothing about these two "abstract" concepts (Figure 1).

More in details, the position regarding the measurement theory can be view as:

- Measurement is an interaction between system and apparatus (or environment).

- Measurements do not uncover some pre-existing physical property of a system. There is no objective property being measured.

- The record or result of a measurement is the only objective property.

If we follow these perspectives, we consider a two-state microsystem whose eigenfunctions are labelled by $\psi_{+}$and $\psi_{-}$. Furthermore, there is a macrosystem apparatus $\phi_{0}$, with eigenfunctions $\phi_{+}$and $\phi_{-}$ corresponding to an output for the microsystem having been in the $\psi_{+}$and $\psi_{-}$states, respectively. Since prior to a measurement we do not know the state of the microsystem, it is a superposition state given by

$$
\psi_{0}=\alpha \psi_{+}+\beta \psi_{-}, \quad|\alpha|^{2}+|\beta|^{2}=1
$$

Now, according to the linearity of Schrödinger's equation, the final state obtained after the interaction of the two systems is

$$
\begin{aligned}
& \Psi_{0}=\left(\alpha \psi_{+}+\beta \psi_{-}\right) \phi_{0} \rightarrow \\
& \Psi_{\text {out }}=\alpha \psi_{+} \phi_{+}+\beta \psi_{-} \phi_{-}
\end{aligned}
$$

where it is assumed that initially the two systems are far apart and do not interact. The state on the far right side of the last equation does not correspond to a definite state for a macrosystem apparatus. In fact, this result would say that the macroscopic apparatus is itself in a superposition of both plus and minus states. Nobody has observed such macroscopic superpositions. This is the measurement problem, since the theory predicts results that are in clear conflict with all observations. It is at this point that the standard program to resolve this problem invokes the reduction of wave packet upon observation, that is,

$$
\alpha \psi_{+} \phi_{+}+\beta \psi_{-} \phi_{-} \rightarrow \begin{cases}\psi_{+} \phi_{+}, & P_{+}=|\alpha|^{2} \\ ; & \psi_{-} \phi_{-}, \\ P_{-}=|\beta|^{2} & .\end{cases}
$$

Various attempts (interpretations) to find reasonable explanation for this reduction are at the heart of the measurement problem.

We will introduce in the next section, three fundamental positions about the concept of physical reality: (i) Einstein, (ii) Rovelli, (iii) Zeilinger.

\section{Physical Reality \\ Einstein Position: Ontological Realism}

Realism, roughly speaking, is the belief that there exists an objective world "out there" independent of our observations. The doctrines of realism are divided into a number of varieties: ontological, semantical, epistemological, axiological, methodological. Ontological studies the nature of reality, especially problems concerning existence, semantical is interested in the relation between language and reality. Epistemological investigates the possibility nature and scope of human knowledge. The 
question of the aims of enquiry is one of the subject of axiology, while methodological studies the best, or most effective means of attaining knowledge. In synthesis:

- (ontological): Which entities are real? Is there a mind-independent world?

- (semantical): Is truth an objective languageworld relation?

- (epistemological): Is knowledge about the world possible?

- (axiological): Is truth one of the aims of enquiry?

- (methodological): What are the best methods for pursuing knowledge?

The Ontological Realism fixed by Einstein (Einstein, 1935) is contained in this fundamental sentence: If, without in any way disturbing a system, we can predict with certainty (i.e., with probability equal to unity) the value of a physical quantity, then there exists an element of physical reality corresponding to this physical quantity.

This was the basic conjecture of the EPR argument with the primary objective to prove the incompleteness of $\mathrm{QM}$. The original paper used entangled pairs of particles states wave, whose function cannot be written as tensor products. Instead of using the quite general configuration, usually is considered an entangled pairs of spin- $1 / 2$ particles that are prepared, following Bohm, in the so-called singlet state that is rotation invariant and given along any vector by:

$$
\Psi\left(x_{1}, x_{2}\right)=\frac{1}{\sqrt{2}}\left(|+\rangle_{1} \otimes|-\rangle_{2}-|-\rangle_{1} \otimes|+\rangle_{2}\right)
$$

The above proposition lead us to analyze two fundamental concepts: (i) the physical quantity and (ii) the physical reality. We have not a univocal definition. In fact, the following affirmation: [...] then there exists an element of physical reality corresponding to this physical quantity. Force us, to consider, an "isomorphism" between two undefined notions: physical reality and physical quantity.

It is quite easy to criticize this relationship. In fact, are there physical quantities with "objective properties", which are independent of any acts of observation or measurement? Moreover, are there elements of physical reality, with a definite values, which do not depend by measurement? We will see that ISSN 13035150
Rovelli's position give us more answers that questions.

\section{Rovelli Position: "Relational" Realism}

Rovelli (1996) departs radically from such strict Einstein realism, the physical reality is taken to be formed by the individual quantum events through which interacting systems (objects) affect one another. Quantum events exist only in interactions and the reality of each quantum event is only relative to the system involved in the interaction. In Relational QM, the preferred observer is abandoned. Indeed, it is a fundamental assumption of this approach that nothing distinguishes, a priori, systems and observers: any physical system provides a potential observer, and physics concerns what can be said about nature on the basis of the information that any physical system can, in principle, have. Different observers can of course exchange information, but we must not forget that such information exchange is itself a quantum mechanical interaction. An exchange of information is therefore a quantum measurement performed by one observing system $A$ upon another observing system $B$.

The physical theory is concerned with relations between physical systems. In particular, it is concerned with the description that observers give about observed systems. Following this hypothesis, all systems are equivalent. Nothing a priori distinguishes observer systems from quantum systems. If the observer $O$ can give a description of the system $S$, then it is also legitimate for an observer $O^{\prime}$ to give a quantum description of the system formed by the observer $\mathrm{O}$. It is rejected any fundamental or metaphysical distinctions as: system/observer, quantum system/classical system, physical system/consciousness. Rovelli assume the existence of an ensemble of systems, each of which can be equivalently considered as an observing system or as an observed system. A system (observing system) may have information about another system (observed system). Information is exchanged via physical interactions.

Rovelli's position, lead us to consider the following epistemological implications:

- (i) rejection of the individual object

- (ii) rejection of individual intrinsic property 
For these reasons, the consequences are: (a) it is not possible to give a definition of the individual object in a spatio-temporal location; (b) it is not possible to characterize the properties of the objects, in order to distinguish from the other ones. In other words, if we adopt the interaction like basic level of the physical reality, we accept the philosophy of the relations.

\section{Zeilinger Position: Epistemological Realism}

The individuality notion was been introduced recently in the radical interpretation of quantum mechanics. The key point is the equivalence between information and individuality (and not between physical quantity and physical reality), this is Zeilinger (2005) view. He put forward an idea which connects the concept of information with the notion of elementary systems. According Zeilinger (1999):

First we note that our description of the physical world is represented by propositions. Any physical object can be described by a set of true propositions. Second, we have knowledge or information about an object only through observations. It does not make any sense to talk about reality without the information about it. Any complex object which is represented by numerous propositions can be decomposed into constituent systems which need fewer propositions to be specified. The process of subdividing reaches its limit when the individual subsystems only represent a single proposition, and such a system is denoted as an elementary system. (qubit of modern quantum physics).

\section{Physical Reality in Nagarjuna Philosophy}

Nagarjuna founded Madhyamika philosophy (Vernette, 2007). At the heart of the Middle Way is the concept of Sunyata. The basic concept of reality in the philosophy of Nagarjuna is that the fundamental reality has no firm core but consists of systems of interacting objects. According to the middle way perspective, based on the notion of emptiness, "phenomena" exist in a relative way, that is, they are empty of any kind of inherent and independent existence. Phenomena are regarded as dependent events existing relationally rather than permanent ISSN 13035150 things, which have their own entity. Nagarjuna middle way perspective emerges as a relational approach, based on the insight of emptiness. Sunyata (emptiness) is the foundation of all things, and it is the basic principle of all phenomena. According to the theory of emptiness, any belief in an objective reality grounded in the assumption of intrinsic, independent existence is untenable. All things and events, whether material, mental or even abstract concepts like time, are devoid of objective, independent existence. To possess such independent, intrinsic existence would imply that things and events are somehow "complete" unto them and are therefore entirely self-contained. This would mean that nothing has the capacity to interact with and exert influence on other phenomena. Moreover, the notion of intrinsic, independent existence is incompatible with the concept of causation.

\section{Concept of Emptiness in other Philosophies.}

The concept of emptiness has been highlighted in many philosophical and mystical works of India in metaphorical and abstract manners. However, this phenomenon has been more logically explained in the Yogic texts by the masters of meditation, who claim of having an experiential dimension of this emptiness concept and relational nature of events. Nagarjuna was himself one such great master of meditation of Buddhism and as quoted, the understanding of emptiness or Madhyamika was revealed to him in the third final stage of meditation (Nagarjuna, 1995). We, thus mention two important citations of mentions of emptiness in Indian Yogic texts, which are same as the description of emptiness in Nagarjunas philosophy, but with distinct portrayals. Another experiential dimension of the relational nature of emptiness is provided by the philosophies of Vihangam Yoga (Prasad, 1989). Founded by His Holiness, Sadguru Sadafal Deo Ji Maharaj, Vihangam Yoga is an ancient meditation technique. This has mentions in ancient Vedic texts. Interestingly in the holy verses of Swarveda, which is the chief text of Vihangam Yoga, several concepts have been mentioned which have vivid descriptions of the concept of emptiness and its relational property. 


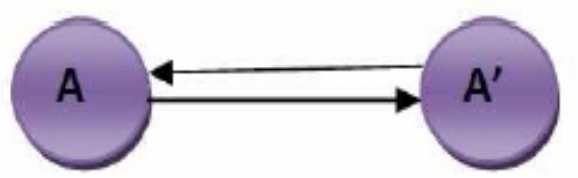

Figure 2. Vihangam Yogic concept of reality of worldly events. If an event is happening to a person $A$, it is actually him observing his own image $A^{\prime}$. Further, $A$ observing $A^{\prime}$ is same as $A^{\prime}$ observing $A$. It is neither $A$ nor $A^{\prime}$ which is real, but the relation between the two (the arrows in above figure). The occurrence of an event can then be described by the relation between $A$ and its own image $A^{\prime}$, which in this philosophy will be the relation that $A$ will bear to $A^{\prime}$ and the vice versa.

We try to highlight some verses of Swarveda, whose descriptions, as we shall see will bring to the fore the concept of emptiness in its relational sense as has been mentioned in Nagarjuna philosophy above.

"The worldly phenomena exist in our relations with them. These events as we observe them are not real, just the relations are real. Events happen in relation to each other, like a person and his own shadow, or like a person and his own image. There is in fact no person or his shadow, or no person or his image, just that they are in relation to each other, which makes the event happen. This relation is again not as simple as we assume. In fact, you looking at your image and your image looking at you are both the same. It is just the specification of the relation between yourself and your image that is real."

So, we find that the author considers events of this world to be unreal and what is real is the relation between entities. The words image and shadow here have been used to specify the unreality of the entity. Thus by saying that the person looking at his own image and the image looking at the person are same, the author specifies that both are actually behaving images of each other and thus both are unreal (or non-existent). Further, he also highlights that the concept of observer and the one being observed are also relational and thus not needed to describe the worldly events.
Emptiness as Relational Properties: the Relational Ontology

What is a property of a quantum object? (Vernette, 2006). According to previous analysis (emptiness/relational), if we adopt the concept of relational as basic level, we accept the philosophy of the relations and we renounce at the possible existence of intrinsic properties. We remember, for instance, that a mathematical model based on the relationist principle accepts that the position of an object can only be defined with respect to other matter. We do not venture in the philosophical implications of the relationalism, as the monism which arm that there are not distinction a priori between physical entities. An important advantage of these approaches is the possibility to eliminate the privileged role of the observer. As we have seen, this is Rovelli's (Rovelli, 1996) approach to quantum mechanics where the founding postulate is the impossibility to talk about properties of systems in the abstract, but only of properties of systems relative to one system (we can never juxtapose properties relative to different systems). Relational quantum mechanics is not the claim that reality is described by the collection of all properties relatives to all systems, rather, reality admits one description per each (observing) system, and any such description is internally consistent. As Einstein's original motivation with EPR was not to question locality, but rather to question the completeness of quantum mechanics, so the relation interpretation can be interpreted as the discovery of the incompleteness of the description of reality that any single observer can give. In this particular sense, relational quantum mechanics can be said to show the "incompleteness" of single-observer Copenhagen interpretation.

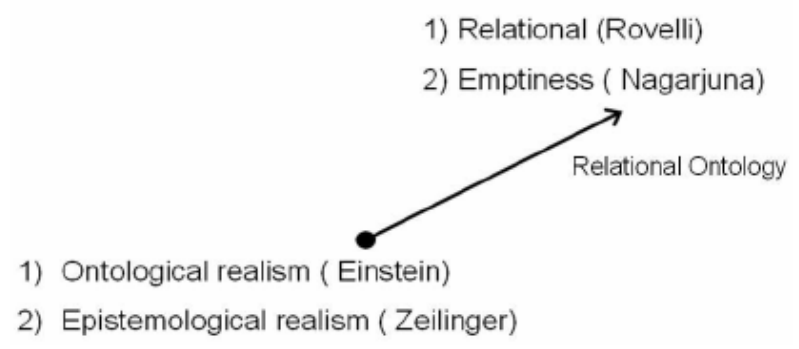

Figure 3. Four positions: starting from 1,2 to 3,4 the role of observer decrease. 


\section{Conclusion}

In this analysis, we suggest to renounce at the ontological and epistemological statute of the physical reality in favour of the relational ontology. The advantages of relational "world" which arise from relational properties are:

1) Any intrinsic property

2) Relational role of observer.

For instance, utilizing a simple metaphor, a camera is a complete tool to "describe" the world? Yes, because we are able to do infinite pictures of the world. In the same way, is the wavefunction is a complete tool for description of underlying physical reality? Yes, because we can do infinite number of measurements with it. In our opinion, the real problem is: the information completeness of a theory is related at the tool or at the world? In other words, the completeness of information belong to the observer or observed? We retain that both possible answers can be seen as idealistic positions. The "tool" is characterized by a relational property. On this basis, we have the possibility to build this new ontology of underlying physical reality: the relational ontology. The basic contention of relational ontology is simply that the relations between entities are ontologically more fundamental than the entities themselves. This contrasts with substantivist ontology in which entities are ontologically primary and relations ontologically derivative. Nagarjuna's philosophy is a relational ontology, because the notion of individuality is considered empty. In the same way, Vihangam Yogic philosophy is relational because it describes all the events in relation to one another.

\section{References}

Bub J. Quantum mechanics is about quantum information. Foundations of Physics, Festschrift issue for James Cushing, 2004.

Einstein A, Podolsky B, Rosen N. Can quantummechanical description of physical reality be considered complete? Phys Rev 1935; 47: 777.

Fuchs CA. Peres: Quantum theory needs no interpretation. Phys Today 2000; 53(3): 70-71.

Fuchs CA. Quantum Mechanics as Quantum Information (and only a little more). arXiv:quant-ph/0205039v1, 2002.

Nagarjuna. The Fundamental Wisdom of the Middle Way. Translated by Jay L. Garfield Published by Oxford University Press US, 1995

Prasad R. Introduction to Vihangam Yoga. Adhyatmic Yantralaya, Garwar, Dist- Ballia, U.P India, 1989.

Rovelli C. Relational quantum mechanics. Intl J Theor Phys 1996;35:1637-1678.

Vernette D, Caponigro M. Physical quantity and Physical reality in Quantum Mechanics: an epistemological path. arXiv:quant-ph/0612036v1, 2006.

Vernette $D$, Tandan $P$, Caponigro $M$. Approach to Physical Reality: a note on Poincare Group and the philosophy of Nagarjuna. arXiv:0704.1665v1, 2007.

Zeilinger A. A foundational principle of Quantum Mechanics. Found of Physics 1999;29: 4.

Zeilinger A. Concept The message of the quantum. Nature 2005; 438: 743. 